\title{
Tolerability and Efficacy of Two Doses of Aerosolized Albuterol in Ventilated Infants with BPD
}

\author{
Natalie Napolitano ${ }^{1}$, Kevin Dysart ${ }^{1}$, Leane Soorikian ${ }^{1}$, Huayan Zhang ${ }^{2}$, Howard Panitch ${ }^{1}$, \\ and Erik Jensen ${ }^{1}$ \\ ${ }^{1}$ The Children's Hospital of Philadelphia \\ ${ }^{2}$ Children's Hospital of Philadelphia
}

September 5, 2020

\begin{abstract}
Rationale: Aerosolized albuterol is widely used, but its safety and efficacy in infants with severe bronchopulmonary dysplasia (sBPD) is not well established. Objectives: To compare the tolerability and efficacy of two dose levels of aerosolized albuterol to saline placebo in infants with sBPD. Methods: Single-center, multiple-crossover trial in 24 ventilated very preterm infants with sBPD. Albuterol $(1.25 \mathrm{mg}, 2.5 \mathrm{mg}$ ) and $3 \mathrm{ml}$ of normal saline were administered every 4 hours during separate 24 -hour treatment periods assigned in random order with a 6-hour washout phase between periods. The primary outcome was the absolute change (post-pre therapy) in expiratory flow at $75 \%$ of exhalation (EF75). Secondary endpoints were changes in ventilator parameters, vital signs, and heart arrhythmia. Results: Average within subject EF75 values improved with each therapy: saline placebo $(+0.45 \mathrm{~L} / \mathrm{min} ? 2.5, \mathrm{p}=0.04), 1.25 \mathrm{mg}$ of albuterol $(+0.70 \mathrm{~L} / \mathrm{min} ? 2.4, \mathrm{p}<0.001)$, and $2.5 \mathrm{mg}$ of albuterol $(+0.38 \mathrm{~L} / \mathrm{min}$ ? 2.4, $\mathrm{p}=0.06)$. However, $1.25 \mathrm{mg}$ of albuterol $(0.26 \mathrm{~L} / \mathrm{min} ; 95 \% \mathrm{CI}-0.19,0.72)$ and $2.5 \mathrm{mg}(-0.10 \mathrm{~L} / \mathrm{min} ; 95 \% \mathrm{CI}$ $-0.77,0.57)$ produced similar changes in EF75 when compared to saline. All secondary outcomes were similar between saline and $1.25 \mathrm{mg}$ of albuterol. Peak inspiratory pressure needed to deliver goal tidal volumes (7.5\% relative decrease, $95 \%$ CI 2.6 , $12.3)$ and heart rate $(6.5 \%$ increase, $95 \%$ CI $2.2,10.8)$ differed significantly between albuterol $2.5 \mathrm{mg}$ and saline. Conclusion: Albuterol at $1.25 \mathrm{mg}$ and $2.5 \mathrm{mg}$, compared to aerosolized saline, did not affect EF75 in infants with sBPD receiving invasive ventilation. Greater improvement in inspiratory pressures with albuterol $2.5 \mathrm{mg}$ suggests benefit, but close heart monitoring is indicated.
\end{abstract}

\section{Introduction}

Bronchopulmonary dysplasia (BPD) is a frequent complication of prematurity manifested by heterogenous clinical phenotypes and incompletely characterized pathophysiology ${ }^{1,2}$. One proposed component of disease pathophysiology in established BPD is hyperresponsiveness of the airway smooth muscle ${ }^{3-5}$. As such, bronchodilators may have therapeutic benefit in some patients ${ }^{6}$.

Aerosolized albuterol is the most commonly used bronchodilator in infants with severe bronchopulmonary dysplasia (sBPD). As many as one-third of infants with sBPD receive this medication during their hospitalization $^{7-11}$. Despite these high rates of use, however, there is a paucity of published data on the safety and efficacy of albuterol in this population. While a small number of studies has examined the efficacy of bronchodilator use for the prevention of BPD, the most recent Cochrane review did not identify any suitable randomized trials on its use in infants with established $\mathrm{BPD}^{12}$. Albuterol is also not approved by the US Food and Drug Administration for use in children under 2 years of age.

With the high, ongoing use of albuterol in infants with sBPD, one immediate concern is identifying an appropriate dose for administration and future study in this population. We undertook the present study to evaluate the short-term tolerability and efficacy of two different doses of aerosolized albuterol (1.25 $\mathrm{mg}$ 
and $2.5 \mathrm{mg}$ ) in very preterm infants with $\mathrm{sBPD}$ receiving invasive mechanical ventilation. We conducted a single-center, multiple cross-over, blinded randomized trial using aerosolized normal saline as a placebo control.

\section{Materials and Methods}

\section{Study Patients}

Eligible infants were born with gestational ages less than 32 weeks, were diagnosed with sBPD at 36 weeks postmenstrual age (PMA) according to the $2001 \mathrm{NIH}$ consensus definition ${ }^{3}$, and were receiving invasive mechanical ventilation at the time of enrollment. Infants were excluded if they were diagnosed with unilateral lung disease, had an endotracheal tube leak $>10 \%$, had prior cardiac arrhythmia, concurrent pulmonary or systemic infection, or were actively treated with high frequency ventilation (HFV), inhaled anticholinergic therapy, or other medication with brochodilatory characteristics (i.e. magnesium sulfate, ketamine, etc.).

Although specific ventilator settings were not mandated for this trial, all subjects were ventilated in a Volume Controlled-Synchronized Intermittent Mandatory Ventilation/Pressure Support (VC-SIMV + PSV) mode with AutoFlow ${ }^{\circledR}$ via the Evita ${ }^{\circledR}$ Infinity ${ }^{\circledR}$ V500 Ventilator (Dräger ${ }^{\circledR}$, Lúbeck, Germany). Our institution utilizes a common strategy for conventional mechanical ventilation within our Chronic Lung Disease Program, involving SIMV-VC/PS with AutoFlow ${ }^{\circledR}$, tidal volumes of $8-12 \mathrm{~mL} / \mathrm{kg}$, PEEP titrated to generate adequate aeration on chest radiograph and minimize patient ventilator asynchrony, and inspiratory time specific to individual patient needs in accordance with analysis of flow-time scalar tracings.

\section{Study Design and Setting}

This interventional, single-center, blinded, placebo-controlled, multiple-crossover randomized trial was designed to examine the short-term tolerability and efficacy of two different doses of aerosolized albuterol: $1.25 \mathrm{mg}$ and $2.5 \mathrm{mg}$. Aerosolized normal saline was used as the placebo control. The trial was conducted in the Neonatal and Infant Intensive Care Unit (N/IICU) at the Children's Hospital of Philadelphia (CHOP) between August 2016 and June 2018. The CHOP N/IICU is a quaternary referral center that primarily cares for outborn infants who require higher levels of intensive care. This study was approved by the Institutional Review Board at CHOP. Parental informed consent was obtained for all enrolled infants.

\section{Intervention, Allocation, and Blinding}

Each aerosolized therapy (albuterol $1.25 \mathrm{mg}$, albuterol $2.5 \mathrm{mg}$, and $3 \mathrm{~mL}$ saline placebo) was administered every 4 hours for up to a total of 6 doses during separate 24-hour treatment periods. A 6-hour washout phase was utilized after each 24-hour treatment period before initiating the subsequent trial therapy. A 6hour monitoring period was used following the final treatment at the end of each infant's trial. The placebo and study drugs were administered in-line to the ventilator circuitry via the Aeroneb ${ }^{\circledR}$ Pro vibrating mesh nebulizer (Aerogen ${ }^{\circledR}$, Galway, Ireland) placed on the inspiratory limb on the dry side of the humidifier, as is the standard practice in our institution.

The order of the three 24-hours treatment periods was randomly assigned in each infant. Briefly, each study participant was assigned to 1 of 6 possible treatment sequences using block randomization (block sizes of 6) in a 1:1 ratio. The randomization sequence was generated using the "ralloc" function in Stata version 13.1. All study randomization and drug preparation procedures were performed by CHOP Investigational Pharmacy to maintain blinding of the study investigators, clinicians, and families. Each study medication dose and saline placebo was drawn up in a $3 \mathrm{ml}$ syringe to an equivalent final volume. The two albuterol doses and saline placebo were not visually distinguishable. All six doses administered during a treatment period were delivered to the participant's bedside in a brown, light protected bag labeled with the appropriate treatment order number $(1,2$, or 3$)$.

\section{Outcomes}

All outcome parameters were measures/recorded prior to administration and 30 minutes after completion of each dose of medication. The primary study outcome was the absolute difference in expiratory flow at $75 \%$ 
of exhalation (EF75), measured directly from the ventilator flow volume loops (Figure 1) 30 minutes post study medication administration compared to immediately prior to administration (post - pre treatment). Measurement of EF75 was accomplished by calculating the exhaled volume at $25 \%$ of the breath (tidal volume $\mathrm{x}$ 0.25) and then recording flow at this point on the expiratory side of the flow volume loop (Figure 1). Measurement of EF75 has not been used in prior studies with mechanically ventilated neonates and was chosen as a measurement that would not require specialized equipment or the need to alter the mechanical ventilator mode or settings to perform measurements. Although the measurement was not performed under conditions of forced exhalation, we reasoned that the measurement would be made over the effort-independent portion of the flow-volume curve, especially in a population of patients with obstructive lung disease. With the administration of a bronchodilator, we anticipated that the EF75 would become higher with small airway dilation.

Secondary outcomes were the proportional change in peak inspiratory pressure (PIP) required to deliver the participant's set tidal volume, minute ventilation, total respiratory rate, blood oxygen saturation level $\left(\mathrm{SpO}_{2}\right)$, heart rate $(\mathrm{HR})$, and the occurrence of any abnormal heart rhythm pre- and post- treatment.

\section{Sample Size Estimation}

To estimate the trial sample size, EF75 values were measured pre- and post- treatment in seven patients clinically prescribed albuterol who otherwise met study inclusion criteria. We observed a mean absolute change in EF75 of $1.3 \mathrm{~L} / \mathrm{min} \pm 0.8$ and powered the trial using a conservative target of approximately half that value, $0.6 \mathrm{~L} / \mathrm{min}$. Sample size estimations were performed in a two-step process. We first calculated the necessary number of participants using a paired t-test for two correlated means. Based on an alpha of 0.05 and correlation of 0.5 , a sample size of at least 21 infants provided [?] $90 \%$ power to detect a mean difference of $0.6 \mathrm{ml} / \mathrm{min}+-0.8$ using a baseline EF75 mean of $2.6 \mathrm{~L} / \mathrm{min}$ and a post-treatment mean of $3.2 \mathrm{~L} / \mathrm{min}$. We then used simulation with 200 replications to model the necessary number of subjects for a generalized linear model based on an estimated correlation of 0.5 between each of the mean EF75 values for the 3 treatment doses. Based on this calculation, a sample size of between 24 and 25 subjects provided approximately $80 \%$ power to detect a mean difference of $0.6 \mathrm{~L} / \mathrm{min}$ between the 3 treatment doses. A sample size of 24 patients was selected to ensure equal representation of the 6 possible treatment ordering schemes.

\section{Statistical Analysis}

Patient demographic and baseline clinical data were summarized with standard descriptive statistics. All continuous study outcome data were summarized using means (+-SD). Post- vs pre-treatment measurements for each drug therapy were compared using paired t-tests. Linear mixed effects models were then used to compare outcome data between the albuterol dose levels and saline placebo. Each model included fixed effects indicator variables for the treatment period (1-3) and within period treatment dose (1-6) to account for any potential temporal effects on the outcome measurements. A random effect (random intercept) for the study participant, robust standard errors, and an unstructured covariance matrix were used in all models. Rates of occurrence for categorical tolerability outcomes were calculated. All statistical analyses were performed using STATA/SE 15.1 (StataCorp, College Station, TX).

Results

Twenty-four infants were enrolled and randomized to receive the 3 study therapies ordered as shown in Figure 2. Two subjects were withdrawn from the trial prior to completing all 3 drugs owing to the development of an exclusion criterion after the trial started (development of airway leak $>10 \%$ and need for HFV). All available study data were used in the analyses. Demographic information for all enrolled subjects is shown in Table 1.

\section{Comparison of Outcome Measures Pre- and Post-Treatment}

Mean values for the study outcome measures recorded immediately prior to the study drug treatment and 30 minutes after treatment completion are summarized in Table 2. Average EF75 values improved with all study therapies, with statistically significant differences observed for saline placebo $(3.9 \mathrm{~L} / \mathrm{min}+-2.0$ vs. 
$4.4 \mathrm{~L} / \mathrm{min}+-2.4, \mathrm{p}=0.04)$ and $1.25 \mathrm{mg}$ of albuterol $(3.8 \mathrm{~L} / \mathrm{min}+-1.7$ vs. $4.5 \mathrm{~L} / \mathrm{min}+-2.2, \mathrm{p}<0.001)$. The differences for $2.5 \mathrm{mg}$ of albuterol approached but did fulfill the cutoff for significance $(3.9 \mathrm{~L} / \mathrm{min}+-1.9$ vs. $4.2 \mathrm{~L} / \mathrm{min}+-2.1, \mathrm{p}=0.06)$. Among the secondary study outcomes, both dose levels of albuterol led to modest but statistically significant reduction in peak inspiratory pressure and increase in minute ventilation but were also associated with a small rise in average heart rate (Table 2). Of the 414 administered drug doses, 31 were associated with a greater than $20 \%$ post-treatment increase in heart rate. Stratified by drug therapy, $14 \%(19 / 132)$ of administered $2.5 \mathrm{mg}$ doses of albuterol were followed by a $>20 \%$ rise in heart rate compared with $6 \%(8 / 144)$ of $1.25 \mathrm{mg}$ albuterol doses and $3 \%(4 / 138)$ of aerosolized saline treatments ( $\mathrm{p}=0.001$ for 3 -way chi square comparison). All heart rate changes were self-limited and did not necessitate subsequent discontinuation of study drug therapy.

\section{Effects of Albuterol Compared to Saline Placebo}

When comparing albuterol to aerosolized saline, neither albuterol dose level significantly increased or decreased average post-therapy EF75 measurements beyond the effects observed for saline placebo (Table 3). The average adjusted differences for the absolute change in EF75 (post - pre-treatment), relative to the level of change with aerosolized saline, were $0.26 \mathrm{~L} / \mathrm{min}(95 \%$ CI $-0.19,0.72)$ for $1.25 \mathrm{mg}$ of albuterol and $-0.10 \mathrm{~L} / \mathrm{min}(95 \% \mathrm{CI}-0.77,0.57)$ for $2.5 \mathrm{mg}$ of albuterol. Similar results were observed when evaluating the proportional change in EF75 [(post-pre)/pre] (Table 3). The 2.5mg of dose of albuterol produced, on average, a $7.5 \%$ (95\% CI 2.6-12.3) greater reduction in the peak inspiratory pressure required to deliver the infants' goal tidal volumes. However, this dose also led on average to $6.5 \%$ (95\% CI 2.2-10.8) greater post-treatment increase in heart rate when compared to aerosolized saline (Table 3).

\section{Discussion}

The objective of this trial was to compare the short-term tolerability and efficacy of two different dose levels of aerosolized albuterol to aerosolized saline placebo in very preterm infants with sBPD receiving invasive mechanical ventilation. Relative to pre-treatment values, albuterol and saline produced significant improvements in EF75. Both dose levels of albuterol also led to modest but significant average post-treatment reductions in peak inspiratory pressure and improvements in minute ventilation. However, when using linear mixed effects models to compare the relative change in outcome measures between albuterol and aerosolized saline, there was no evidence of net benefit or harm with either albuterol dose level for the primary outcome of EF75. The 2.5mg dose of albuterol was more effective than saline placebo for the outcome of post-treatment peak inspiratory pressure, but with a small average net increase in heart rate. Collectively, these trial data suggest that aerosolized albuterol and saline can positively affect respiratory dynamics in ventilated very preterm infants with sBPD. A $2.5 \mathrm{mg}$ dose of albuterol may provide a modest respiratory advantage over the other two studied therapies, but clinicians should monitor changes in heart rate if administering this dose.

To our knowledge, this is the first randomized, placebo controlled trial to evaluate aerosolized bronchodilator therapy for the treatment of established sBPD.

Prior trials have examined inhaled bronchodilators in ventilated preterm infants at high risk of developing $\mathrm{BPD}^{13-18}$. Although most of these studies used an alternative drug therapy or compared the efficacy of different delivery devices rather than a placebo control, they collectively show post-treatment improvements in respiratory mechanics and oxygenation with inhaled bronchodilator therapy in ventilated preterm infants ${ }^{13-18}$. More recently, several observational studies have examined the use of aerosolized albuterol in preterm infants with evolving or established $\mathrm{sBPD}^{5,9,10,19}$. In a retrospective analysis of pulmonary function testing (PFT) data, Morrow et al. assessed the response to bronchodilator therapy in infants with evolving BPD using a single breath occlusion technique ${ }^{19}$. Fifty-two percent of infants studied had a reduction in respiratory system resistance of $10 \%$ or greater, $13 \%$ had a compliance reduction of $<10 \%$, while $35 \%$ had no apparent response to bronchodilator administration ${ }^{19}$. There were no significant differences in respiratory system compliance measurements for any infants ${ }^{19}$. Shepherd et al. also observed heterogeneity in the bronchodilator responsiveness in a retrospective cohort of infants with established sBPD, noting greater efficacy associated with predominately obstructive rather than restrictive lung disease ${ }^{9}$. Potentially consistent with 
the findings of Morrow et al, our observation of a post-treatment reduction in PIP may be the result of a reduction in pulmonary resistance with aerosolized bronchodilator therapy.

We chose expiratory flow at $75 \%$ of the exhaled volume as the primary means to assess bronchodilator responsiveness in this study population. We hypothesized that relaxation of airway smooth muscle would increase expiratory flow from $0-75 \%$ of the tidal volume and produce a net increase in EF75 indicating a positive bronchodilatory effect. As a secondary outcome, we examined changes in peak inspiratory pressure. Since we did not change ventilator settings after aerosol administration, we propose that peak inspiratory pressure during volume guarantee ventilation is a reasonable surrogate measure of changes in airway resistance. Of note, previous studies have used alternative methods to assess bronchodilator responsiveness in infants and children ${ }^{13,20-22}$. However, these methods require manipulation and/or interruption of the ventilators settings and pattern intern potentially changing the pulmonary mechanics from those of the primary ventilator strategy. Measurement of tidal mechanics using ventilator output signals requires a change in ventilator support to constant flow without pressure regulation, and imposition of an inspiratory hold in a passively ventilated patient ${ }^{21,22}$. Other available methods require the use of specialized equipment to perform brief occlusions ${ }^{13,20}$. We purposely chose a measure that not need specialized equipment or require a change to the patients' mechanical ventilation settings. This enabled us to use an outcome measure that could be easily reproduced in any NICU regardless of research resources (equipment or personnel) and would not require multiple disconnections of the ventilator circuitry which are typically not well tolerated in this patient population.

The lack of an apparent benefit of albuterol relative to saline placebo for our primary outcome of EF75 may be the result of a positive treatment effect of normal saline. Prior studies have shown benefit with aerosolized saline in conditions such as chronic obstructive pulmonary disease and bronchiolitis ${ }^{23,24}$. Aerosolized saline may increase hydration of the mucocillary bed, improve ciliary function and mucous clearance, and thereby decrease airway resistance ${ }^{25,26}$. The observed improvements in EF75 after nebulized saline suggest airway surface dehydration and resultant ciliary dysfunction could exist in the sBDP population. Despite a potential treatment effect, however, our use of normal saline a placebo is consistent with multiple other randomized controlled trials of bronchodilators ${ }^{24}$. Moreover, normal saline is present in albuterol solutions used for nebulization. Thus, our use of normal saline simply removed the active drug from the nebulized solution. An alternative study design could employ a sham treatment without an actual placebo. This may overcome the apparent treatment effects of aerosolized saline, but could negatively impact the masking of clinicians and investigators to the assigned therapy.

Traditionally at our facility, the dose of nebulized medications is selected according to patient weight. However, this practice contradicts to the results of aerosolization studies, which show that alveolar drug delivery depends on breathing patterns and is largely independent of patient weight ${ }^{27-29}$. High respiratory rates and small tidal volumes effect deposition because less of the medication is drawn into the lungs and the change in respiratory pattern as children age into adults results in greater deposition of medication ${ }^{27-29}$. This change in pattern of deposition results in self-regulation of dose as breathing pattern changes. Additionally, we commonly administer aerosolized medications through an artificial airway during mechanical ventilation. In vitro studies of this route of administration show that only $12 \%-14 \%$ of the total drug dose is delivered to the distal airways under these conditions ${ }^{30,31}$.

There are several limitations to this study. First, this was a single center trial and may not be generalizable to populations in other centers. Although the number of enrolled infants was selected by an a priori sample size estimation, the overall number of participants was relatively small and larger studies are needed to confirm the study findings. Lastly, the primary outcome utilized in this trial is not an established indicator of bronchodilator response in mechanically ventilated infants, but our secondary outcomes, including measurement of PIP, have been used in prior studies of bronchodilator responsiveness in mechanically ventilated subjects $^{20,33,34}$. 


\section{Conclusion}

Relative to measured pre-treatment values, aerosolized albuterol and saline produced post-treatment improvements in the primary study outcome of EF75 in preterm infants with sBPD receiving invasive mechanical ventilation. However, albuterol administered at $1.25 \mathrm{mg}$ or $2.5 \mathrm{mg}$ did not significantly improve EF75 when compared to saline placebo. Relative to saline placebo, albuterol at a dose of $2.5 \mathrm{mg}$ did result in a modest decrease in the PIP required to deliver the set tidal volume, suggesting a possible benefit for reducing airway resistance. Notably, some infants experienced self-limited increases in heart rate after administration of this higher dose suggesting close monitoring of vital signs is indicated.

\section{References}

1. Lui K, Lee SK, Kusuda S, Adams M, Vento M, Reichman B, Darlow BA, Lehtonen L, Modi N, Norman $\mathrm{M}$, et al. Trends in outcomes for neonates born very preterm and very low birth weight in 11 highincome countries. J Pediatr, 215 (2019), pp. 32-40.e14

2. Wu KY, Jensen EA, White AM, Wang Y, Biko DM, Nilan K, Fraga MV, Mercer-Rosa L, Zhang H, Kirpalani H. Characterization of disease phenotype in very preterm infants with severe bronchopulmonary dysplasia. Am J Respir Crit Care Med. 2020;201(11):1398-1406.

3. Jobe AH, Bancalari E. Bronchopulmonary dysplasia. Am J Respir Crit Care Med 2001;163(7):17231729.

4. Walsh MC, Szefler S, Davis J, Allen M, Van Marter L, Abman S, Blackmon L, Jobe A. Summary Proceedings from the bronchopulmonary dysplasia group. Pediatrics 2006;117(3 Pt 2):S52-S56.

5. Shepherd EG, Clouse BJ, Hasenstab KA, Sitaram S, Malleske DT, Nelin LD, Jabcherla SR. Infant pulmonary function testing and phenotypes in severe bronchopulmonary dysplasia. Pediatrics 2018;141(5):e20173350 [epub ahead of print]

6. Smyth JA, Tabachnik E, Duncan WJ, Reilly BJ, Levison H. Pulmonary function and bronchial hyperreactivity in long-term survivors of bronchopulmonary dysplasia. Pediatrics 1981;68(3):336-340.

7. Clark RH, Bloom BT, Spitzer AB, Gerstmann DR. Reported medication use in the neonatal intensive care unit: data from a large national data set. Pediatric 2006; 117(6):1979-1987.

8. Allen J, Zwerdling R, Ehrenkranz R, Gaultier C, Geggel R, Greenough A, Kleinman R, Klijanowicz A, Martinez F, Ozdemir A, Et al. Statement on the care of the child with chronic lung disease of infancy and childhood. Am J Respir Crit Care Med 2003; 168(3):356-396.

9. Slaughter JL, Stenger MR, Reagan PB, Jadcherla SR. Inhaled bronchodilator use for infants with bronchopulmonary dysplasia. J Perinatology 2015;35:61-66.

10. Guaman MC, Gien J, Baker CD, Zhang H, Austin ED, Collaco JM. Point prevalence, clinical characteristics, and treatment variation for infants with severe bronchopulmonary dysplasia. Am J Perinatol 2015;32(10):960-967.

11. Bamat NA, Kirpalani H, Feudtner C, Jensen EA, Laughon MM, Zhang H, Monk HM, Passarella M, Lorch SA. Medication use in infants with severe bronchopulmonary dysplasia admitted to United States children's Hospitals. J Perinatol 2019;39(9):1291-1299.

12. Ng Gy, da Silca O, Ohlsson A. Bronchodilators for the prevention and treatment of chronic lung disease in preterm infants. Cochrane Database Syst Rev. 2012; 6.

13. Fayon M, Tayara N, Germain C, Choukroum ML, De La Roque ED, Chene G, Breilh D, Marthan R, Demarquez JL. Efficacy and tolerance of high-dose inhaled ipratropium bromide vs terbutaline in intubated premature human neonates. Neonatology 2007;91:167-173.

14. Lee H, Arnon S, Silverman M. Bronchodilator aerosol administered by metered dose inhaler and spacer in subacute neonatal respiratory distress syndrome. Arch Dis in Childhood 1994;70:218-22.

15. Pfenninge J, Aebi C. Respiratory response to salbutamol (albuterol) in ventilator-dependent infants with chronic lung disease: pressurised aerosol delivery versus intravenous injection. Intensive Care Medicine 1993;19:251-5.

16. Rotschild A, Solimano A, Puterman M, Smyth J, Sharma A, Albersheim S. Increased compliance in 
response to salbutamol in premature infants with developing bronchopulmonary dysplasia. J Pediatrics 1989;115:984-91.

17. Sivakumar D, Bosque E, Goldman SL. Bronchodilator delivered by metered dose inhaler and spacer improves respiratory system compliance more than nebulizer-delivered bronchodilator in ventilated premature infants. Pediatr Pulm 1999;27:208-12.

18. Wilkie RA, Bryan MH. Effect of bronchodilators on airway resistance in ventilator-dependent neonates with chronic lung disease. J Pediatrics 1987;111:278-83.

19. Morrow Dk, Schilling D, McEvoy CT. Response to bronchodilators in very preterm infants with evolving bronchopulmonary dysplasia. Res Rep Neonatol 2015; 5:113-117.

20. Levin DL, Garg A, Hall LJ, Slogic S, Jarvis JD, Leiter JC. A prospective randomized controlled blinded study of three bronchodilators in infants with respiratory syncytial virus bronchiolitis on mechanical ventilation. Pediatr Crit Care Med 2008;9:598-604.

21. Carrol CL, Sala K, Zucker AR, Schramm CM. Pulmonary mechanics following albuterol therapy I mechanically ventilated infants with bronchiolitis. J Asthma 2012;49(7):688-296.

22. Schramm CM, Sala KA, Carroll CL. Clinical examination does not predict response to albuterol in ventilated infants with bronchiolitis. Pediatr Crit Care Med 2017;18(1):e18-e23.

23. Khan SY, O'Driscoll BR. Is nebulized saline a placebo in COPD? BMC Pulm Med . 2004;4:9.

24. House SA, Gadomski AM, Ralston SL. Evaluating the placebo status of nebulized normal saline in patients with acute viral bronchiolitis: A systematic review and meta-analysis. JAMA Pediatr 2020;174(3):250-259.

25. Van der Schans CP. Bronchial mucus transport. Resp Care 2007;52(9):1150-1158.

26. Rogers DF. Mucoactive agents for airway mucus hypersecretory diseases. Resp Care 2007;52(9):11761193.

27. Schuepp KG, Straub D, Moller A, Wildhaber JH. Deposition of aerosols in infants and children. Journal of Aerosol Medicine 2004;17(2):153-156.

28. Amirav I, Newhouse MT. Deposition of small particles in the developing lung. Pediatric Respiratory Reviews 2012;13:73-78.

29. Fink JB. Aerosol delivery to ventilated infants and pediatric patients. Respiratory Care 2004;49(6):653665.

30. Dubus JC, Vecellio L, de Monte M, Fink JB, Grimbert D, Montharu J, Valat C, Behan N, Diot P. Aerosol deposition in neonatal ventilation. Pediatric Res 2005;58(1):10-4.

31. Berlinski A, Willis JR. Effect of tidal volume and nebulizer type and position on albuterol delivery in a pediatric model of mechanical ventilation. Respiratory Care 2015;60(10):1424-1430.

32. Johnston DA, Gilmore TW, Gosselin KP. A comparison of metered-dose inhaled albuterol vs endotracheal liquid bolus albuterol for treatment of bronchoconstriction. Resp Care 2015; 60(5)627-635.

33. ElHansy MHE, Boules ME, El Essawy AFM, Al-Kholy MB, Abdelrahman MM, Said ASA, Hussein RRS, Abdelrahim ME. Inhaled salbutamol dose delivered by jet nebulizer, vibrating mesh nebulizer and metered dose inhaler with spacer during invasive mechanical ventilation. Pulm Pharmacol Ther 2017;45:159-163.

\section{Hosted file}

ImageLegends_29August2020.docx available at https://authorea.com/users/354995/articles/ 478427-tolerability-and-efficacy-of-two-doses-of-aerosolized-albuterol-in-ventilatedinfants-with-bpd

\section{Hosted file}

Tables_Final_29August2020.docx available at https://authorea.com/users/354995/articles/ 478427-tolerability-and-efficacy-of-two-doses-of-aerosolized-albuterol-in-ventilatedinfants-with-bpd 

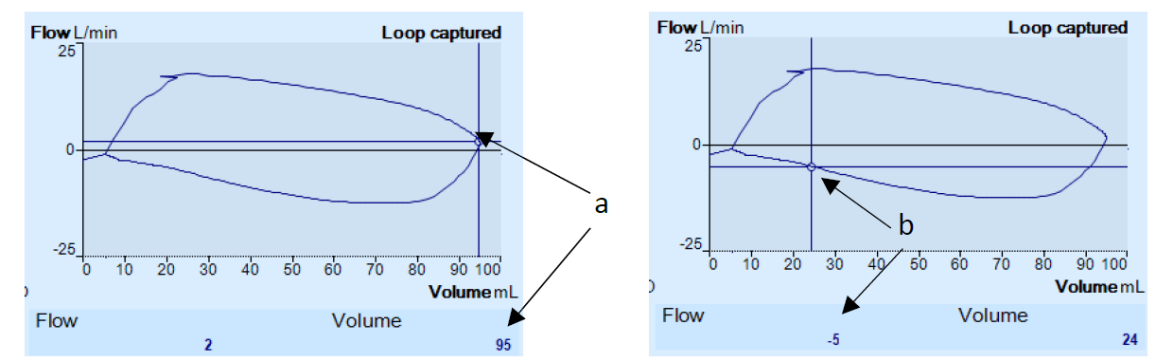

Assessed for Eligibility $(\mathrm{n}=108)$

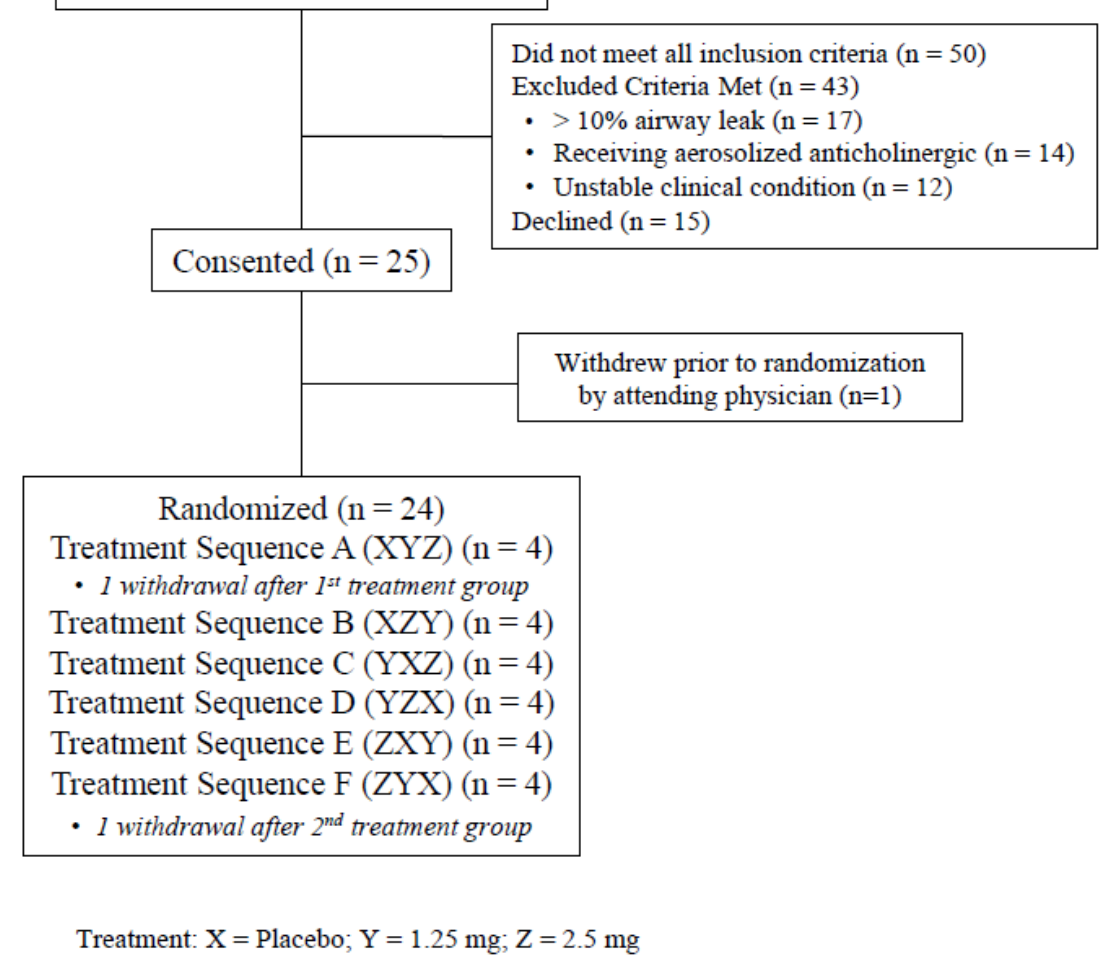

